consequences were encountered by $5 / 11$ (45\%) (3 did not answer). Consequences included increased drug dealing in the area and patients swapping vouchers for drugs.

Of those who agreed with incentives, $71 \%$ felt shopping or taxi vouchers would be preferable to cash. Appropriate value was split; $41 \%$ felt it should be $\leq £ 10,27 \%>£ 10 \leq £ 40$ and $32 \%>£ 40 \leq £ 50$.

Timing of incentivise could be at initial testing (preference for $8 \%$ ), starting or completing treatment (preference for $21 \%$ $\& 25 \%$ respectively) or at all of these opportunities (46\%).

There was no clear consensus on who would be best placed to give the incentive; $35 \%$ felt drug \& alcohol services, $15 \%$ nurse specialist, $15 \%$ peer support worker, 5\% pharmacist, $2.5 \%$ doctor and $27.5 \%$ felt any of these could provide it (respondents could select more than one option).

Respondents were asked how comfortable they felt patients with HCV would be with offering incentivisation (very uncomfortable $=1$, very comfortable $=5$ ). $68.9 \%$ of respondents felt patients with HCV would be comfortable or very comfortable with incentives.

Conclusions There was no clear consensus on incentivisation within these two HCV ODNs. Where offered, incentives have been felt to promote a rise in engagement, but alongside negative consequences. Vouchers were deemed preferable to cash. The survey was useful in gauging current attitudes as ODNs seek bold and innovative ways to test and treat HCV in working towards eradication. The impact of incentivisation where used should be carefully assessed to look for efficacy and any negative consequences.

\section{PWE-30 USE OF A DISCHARGE BUNDLE CAN IMPROVE POST- ADMISSION CARE FOR PATIENTS WITH DECOMPENSATED CIRRHOSIS}

Sonia Moteea*, Neil Gordon, Lynsey Corless. Hull University Teaching Hospitals NHS Trust, Hull, UK

\subsection{6/gutjnl-2021-BSG.220}

Introduction Decompensated Chronic Liver Disease (DCLD) is a medical emergency with high morbidity and mortality. Appropriate follow-up and monitoring can reduce readmissions and improve both outcomes and quality of life. It is vital that discharge planning and documentation is of a high standard to ensure key aspects of post-admission follow up are arranged and communicated to the patient and primary care team. The BSG Decompensated Cirrhosis Discharge Bundle (https://www.bsg.org.uk/clinical-resource/decompensated-cirrhosis-discharge-bundle/) was developed to support high-quality discharge planning, and we sought to evaluate the use of the bundle in our hospital.

Methods A retrospective audit of case notes and discharge summaries was conducted of all patients discharged from the gastroenterology ward with a diagnosis of DCLD over 3 months. This data was used to establish a baseline for completion/documentation of each item in the bundle checklist. The bundle was then implemented, and a further 40 DCLD discharges subsequently reviewed against the same criteria, followed by analysis of pre- vs post-bundle results.

Results The baseline cohort comprised 40 patients (21 male, 19 female; median age 60 [38-83]), with a further 40 patients included in the second cycle ( 25 male, 15 female; median age 45 [33-65]). Hepatic encephalopathy $(63 \%, n=25)$ and ascites
$(70 \%, \mathrm{n}=28)$ were commonest reasons for admission. Introduction of the bundle led to improvements in several areas. $80 \%$ of appropriate patients had an out-patient paracentesis procedure booked prior to discharge after bundle introduction, compared to just $7.7 \%$; $p<0.001$ ) beforehand. Likewise, variceal screening endoscopy was arranged for $50 \%$ post-bundle (27.5\% pre-bundle; $p=0.21$ ), and more of those with recently identified varices were appropriately discharged on primary prophylaxis $(50 \%$ vs $37.5 \% ; p=0.26)$. Patients admitted with hepatic encephalopathy were more likely to be discharged with appropriate lactulose $(93 \%$ vs $40 \% ; p<0.0001)$ and Rifaximin (63\% vs $22.5 \%$; $p<0.0001)$. Those with alcohol related liver disease had improved rates of planned community follow-up $(83 \%$ vs $11 \% ; p<0.0001)$ and documented need for abstinence $(100 \%$ vs $30 \%$; $p<0.0001)$. Finally, communication was also improved, both for advice to primary care about renal function (documented in $70 \%$ vs $8.8 \% ; p<0.001$ ), and explanation of the need for specific medications $(50 \%$ vs $12.5 \% ; p<0.0001)$.

Conclusion Implementation of the discharge bundle led to improved discharge planning and documentation, justifying the continued use of the checklist for patients hospitalized with DCLD.

\section{PWE-31 USE OF NON-SELECTIVE BETA-BLOCKERS (NSBB) IS NOT DETRIMENTAL IN CIRRHOTIC PATIENTS WITH SEVERE ASCITES}

Chaonan Dong ${ }^{*}$, Alice Weidner, Rohit Sinha. Sunderland Royal Hospital, Sunderland, UK

\subsection{6/gutjnl-2021-BSG.221}

Introduction The use of non-selective beta-blockers (NSBB) is the cornerstone in the treatment of portal hypertension. Despite convincing data, scepticism surrounding safety of NSBB, particularly in severe ascites remains. We aim to study the survival outcome in patients with liver cirrhosis and severe ascites on NSBB.

Methods This was a single-centre retrospective study of 131 patients with liver cirrhosis and ascites who presented consecutively from January 2014 to December 2019 by attending nurse-led large volume paracentesis (LVP) service at the Sunderland Royal Hospital. Data was obtained from patient notes using Meditech $^{\text {TM }}$ patient database. Survival was calculated from the date when first LVP was performed. Two time-varying covariates, Transjugular Intrahepatic Porto-Systemic Shunt (TIPSS) and liver transplant, which occurred during the follow-up period were included in the adjusted analysis. Cox regression was used to compare the survival times in the two groups, both before and after adjustments for potential cofounders.

Results Patient characteristics and other observed variables were comparable in the NSBB $(n=47)$ and No-NSBB $(n=84)$ group. Alcohol was the commonest aetiology of cirrhosis and comparable $(p=0.72)$ across groups, $87 \%$ and $89 \%$ of NSBB and no-NSBB group respectively. $66 \%$ and $64 \%$ of patients continued to drink alcohol in the NSBB and no-NSBB group $(p=0.85)$. Refractory ascites was present and comparable $(p=0.76)$ in $68 \%$ and $65 \%$ of the NSBB and no-NSBB group respectively. The frequency of LVP was comparable between the groups $(p=0.13)$. The median MELD was comparable $(p=0.65)$ at $15.2[11.8,18.7]$ and $15.0[10.3,18.4]$ in the NSBB and no-NSBB groups. There was no statistically 


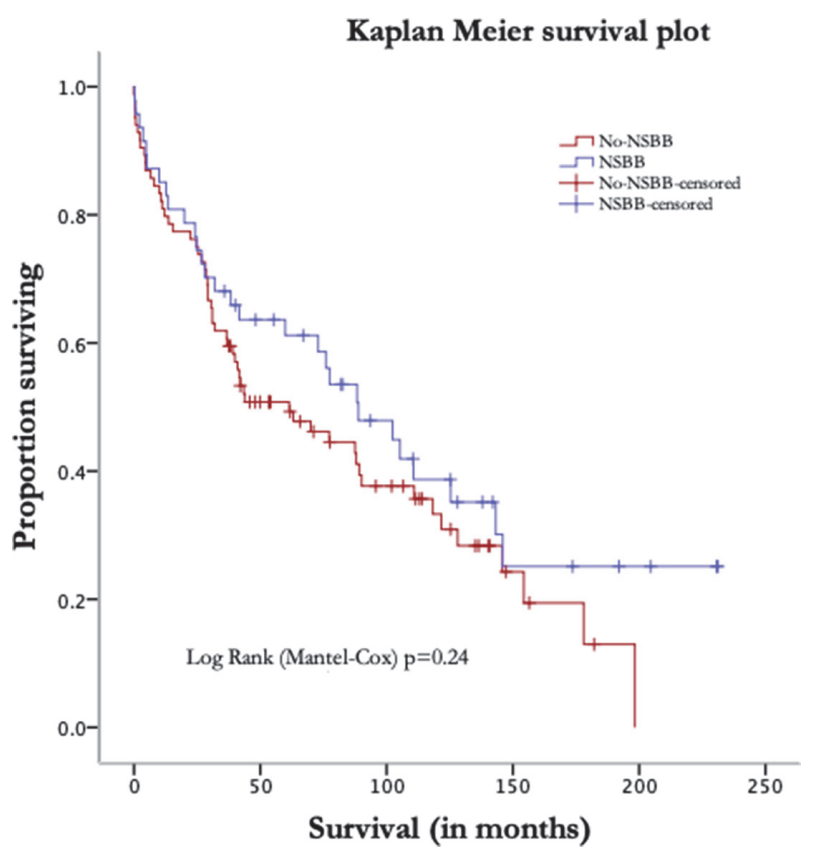

Abstract PWE-31 Figure 1 A graphical illustration of the survival times in the two groups is shown in the Kaplan-Meier plot

significant difference in survival between the two groups $(p=0.24)$ as seen in figure 1 . The adjusted hazard risk analysis (0.75 [95\%CI 0.51, 1.11], $p=0.15)$ suggested weak evidence of a difference in survival between the two groups. Median survival times were slightly longer in the NSBB group, 88.8 months (95\%CI 53.2,124.4) compared with No-NSBB group 61.4 months $(95 \%$ CI 26.6, 96.2); and the risk of death at any time lower. TIPSS, was performed in 6 patients of NoNSBB and 4 with NSBB group. Two patients received liver transplant, both in the no-NSBB group. Diuretic intractable ascites $(p=0.06)$, MELD $(p=0.018)$ and frequency LVP sessions $(p<0.001)$ were all independent predictors of mortality. Conclusions Our study demonstrates that NSBB is safe in cirrhotic patients with severe ascites, and it is not associated with detrimental outcome. There is need for a randomised controlled trial to draw conclusive evidence.

\section{PWE-32 THE EFFECTIVENESS OF HEPATOCELLULAR CARCINOMA SURVEILLANCE DURING COVID-19}

${ }^{1}$ William Cunliffe*, 1,2Thomas Pembroke. ${ }^{1}$ Cardiff Medical School, Cardiff, UK; ${ }^{2}$ Department of Gastroenterology and Hepatology, University Hospital of Wales, Cardiff, UK

\subsection{6/gutjnl-2021-BSG.222}

Introduction The incidence of hepatocellular carcinoma (HCC) in Wales is rising. We aimed to evaluate the impact of the COVID-19 pandemic on the South Wales HCC multi-disciplinary meeting (MDM) referrals and patient management.

Methods Baseline patient characteristics at MDM and treatment information was collated for index HCC discussion from the electronic health record. The year of the COVID period (CP) $01 / 03 / 2020-28 / 2 / 2021$, was compared to the prepandemic period (PP) 01/03/2018-29/02/2020. To determine significance, a $\mathrm{Chi}^{2}$ analysis or Fisher's Exact test were used where appropriate.

Results There was a 3-fold increase in index HCC diagnoses in South Wales MDM between 2002 and 2018. 803 patients were discussed from March 2018-21; follow up (372) and non-HCC (90) cases were excluded. There were $245 \mathrm{PP}$ and 96 CP index HCC diagnoses; a $22 \%$ reduction in annual index HCC diagnoses in the COVID period. The absolute number of HCCs detected by surveillance remained consistent at 33 per annum in $\mathrm{PP}$ and $\mathrm{CP}$ periods $(27 \% \mathrm{v} 34 \%$ respectively). During CP the proportion of patients offered best supportive care and undergoing further investigation increased (47 to $56 \%$ and 1.2 to $13.5 \%$ respectively) whilst all anti-cancer interventions fell (figure 1): ablation/surgery $(22$ to $15.6 \%)$, chemo-embolisation (21.2 to $11.5 \%)$ and systemic anti-cancer therapies (8.2 to $3.1 \%, \mathrm{p}<0.0001)$. However, during $\mathrm{CP}$ a greater proportion of those on HCC surveillance received curative therapies compared to HCC detected in individuals with cirrhosis outside of screening (27.3 vs. $8.5 \%$, $\mathrm{p}=0.03$ ). Median time from point of suspicion of HCC to

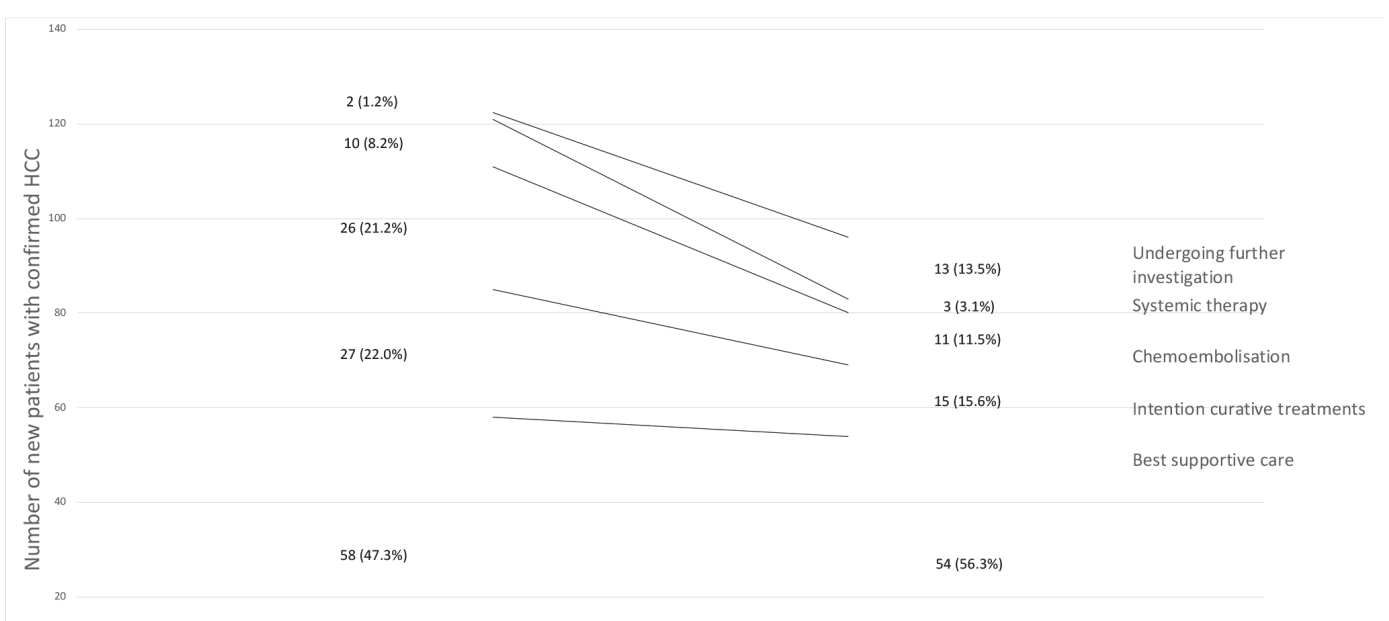

Pre-pandemic period 12-month average

COVID period

Abstract PWE-32 Figure 1 Treatment outcomes for HCC MDM discussion in South Wales in the Pre-pandemic and COVID periods 